\title{
Application and Development of Functional Magnetic Nanomaterials in Food Quality and Safety Inspection
}

\author{
Rui $\mathrm{Su}^{1, *}$ \\ ${ }^{1}$ College of Biological Sciences and Biotechnology, Beijing Forestry University, 100083, China
}

\begin{abstract}
At present, people are paying more and more attention to the accuracy, convenience, and efficiency of food quality and safety testing. However, many traditional detection methods have some problems such as inconvenient operation, interference factors, and long detection time. Therefore, functionalized magnetic nanomaterials with higher selectivity and sensitivity are widely used in food detection, such as pesticides, veterinary drugs, heavy metals, additives, and synthetic pigments, pathogenic bacteria, and mycotoxins. In this paper, the practical application of functionalized magnetic nanomaterials and their related detection technologies are reviewed, and their future development direction is discussed. Functional magnetic nanomaterials can solve some problems that exist in magnetic nanomaterials to a great extent, making detection more efficient, economical, accurate, rapid and convenient. However, in the future, people still need to further study more stable and efficient magnetic nanomaterials to cope with various complex detection situations.
\end{abstract}

\section{Introduction}

Food quality and safety is a big problem related to social stability and harmonious development. However, in recent years, the melamine, Sudan red, and other problematic food incidents in China have triggered public vigilance and nervousness about food safety issues. Meanwhile, food industry personnel are stepping up to strengthen testing technology and strictly revising relevant laws and regulations. At present, the widely used detection methods in China are still relatively traditional, such as enzyme-linked immunosorbent assay (ELISA), thin-layer chromatography (TLC), gas chromatography (GC), high-performance liquid chromatography (HPLC) and GC-MS ${ }^{[1]}$. These technologies also have some obvious problems such as longer detection time, inconvenient operation, and more interference factors. Therefore, there is an urgent need to develop a detection means not only more rapid, economic, sensitive, and accurate, but also anti-interference and easy to operate.

How to break through the defect of detection brought by traditional technology? In nature, turtles can travel tens of thousands of kilometers to return to the beach of their birth with great precision, relying on nano-magnetic materials in their heads. Food quality and safety testing often require more accurate and sensitive detection technology. Therefore, this paper explores the application of magnetic nanomaterials that can be precisely positioned in food quality and safety detection, and summarizes the preparation methods of magnetic nanomaterials. The techniques for applying magnetic nanomaterials as well as the application of magnetic nanomaterials in the detection of different substances are discussed. It can be concluded from pieces of literature that the

\footnotetext{
* Corresponding author: 1070059339@qq.com
}

functionalization of magnetic nanomaterials needs to advance towards the direction of higher selectivity, high flux, and high sensitivity. In terms of technology, more convenient in situ real-time monitoring is realized, and more miniaturized, automated, high-throughput, and online detection methods are developed by combining functional magnetic nanomaterials technology with various analytical technologies.

\section{Overview of magnetic nanomaterials}

Nanomaterials is a scaling concept that refers to materials in which at least one dimension of a three-dimensional space is nanometer-sized $(1-100 \mathrm{~nm})$ or consists of them as basic units. Inorganic magnetic nanomaterials such as $\mathrm{Fe}_{3} \mathrm{O}_{4}$ are mainly used in food analysis. At present, the preparation methods of magnetic nanoparticles mainly include the coprecipitation method, high-temperature decomposition method, microemulsion method, and reverse micelle method.

Coprecipitation method is mainly led by $\mathrm{Fe}^{2+}+2 \mathrm{Fe}^{3+}$ $+8 \mathrm{OH}^{-} \rightarrow \mathrm{Fe}_{3} \mathrm{O}_{4}+4 \mathrm{H}_{2} \mathrm{O}^{[2]}$. The method is now widely used. High-temperature decomposition method uses a high-temperature organic solvent with a high boiling point, and also contains a surfactant. The reaction raw material is injected rapidly to make organic metal compound decomposed by heating, making rapid nucleation, and then we get magnetic nanoparticles. In microemulsion method and reverse micelle method, microemulsions and reverse micelles which are formed from ternaries of water, oil, and surfactant are used as a reaction space ${ }^{[3]}$. Surfactants can effectively control particle size and make particles had better solubility and dispersion. 
Table 1. Comparison of preparation methods of magnetic nanoparticles

\begin{tabular}{|c|c|c|}
\hline Coprecipitation method & Advantages & Disadvantages \\
\hline $\begin{array}{c}\text { High purity, small particle size, uniform } \\
\text { particle size, and good dispersibility } \\
\text { were obtained. The operation is simple } \\
\text { and the reaction condition is mild. }\end{array}$ & $\begin{array}{c}\text { There are many influencing factors in the } \\
\text { reaction, so it is necessary to control the } \\
\text { product composition and prevent the } \\
\text { nanoparticles from agglomerating. }\end{array}$ \\
\hline $\begin{array}{c}\text { High-temperature decomposition } \\
\text { method }\end{array}$ & $\begin{array}{c}\text { High particle purity, narrow particle } \\
\text { size distribution, and controllable size } \\
\text { morphology }\end{array}$ & $\begin{array}{c}\text { There are some limitations, like } \\
\text { providing high-temperature solvents. }\end{array}$ \\
\hline $\begin{array}{c}\text { Microemulsion method and reverse } \\
\text { micelle method }\end{array}$ & $\begin{array}{c}\text { The particle size distribution and } \\
\text { morphology are well controlled. }\end{array}$ & $\begin{array}{c}\text { Particle crystallinity and magnetic } \\
\text { response need to be improved }\end{array}$ \\
\hline
\end{tabular}

Magnetic nanoparticles can display strong magnetic and good magnetic fine, the crystal of magnetic induction in the external magnetic field, without which there is no magnetic induction ${ }^{[1]}$. This property is called superparamagnetism, which optimizes the enrichment and separation of target substances in food analysis. Table 1 summarizes the above methods for preparing magnetic nanoparticles. Magnetic nanoparticles have simple preparation, good superparamagnetism, high specific surface area, and easy modification. However, when $\mathrm{Fe}_{3} \mathrm{O}_{4}$ and $\gamma-\mathrm{Fe}_{2} \mathrm{O}_{3}$ are used as adsorbents only, particles tend to agglomerate, resulting in poor adsorption selectivity and reduced enrichment effect. To make magnetic nanoparticles widely used in food safety testing analysis, we have to further research in the future to make a more simple and feasible preparation of the process, quickly get narrower particle size distribution, high purity,

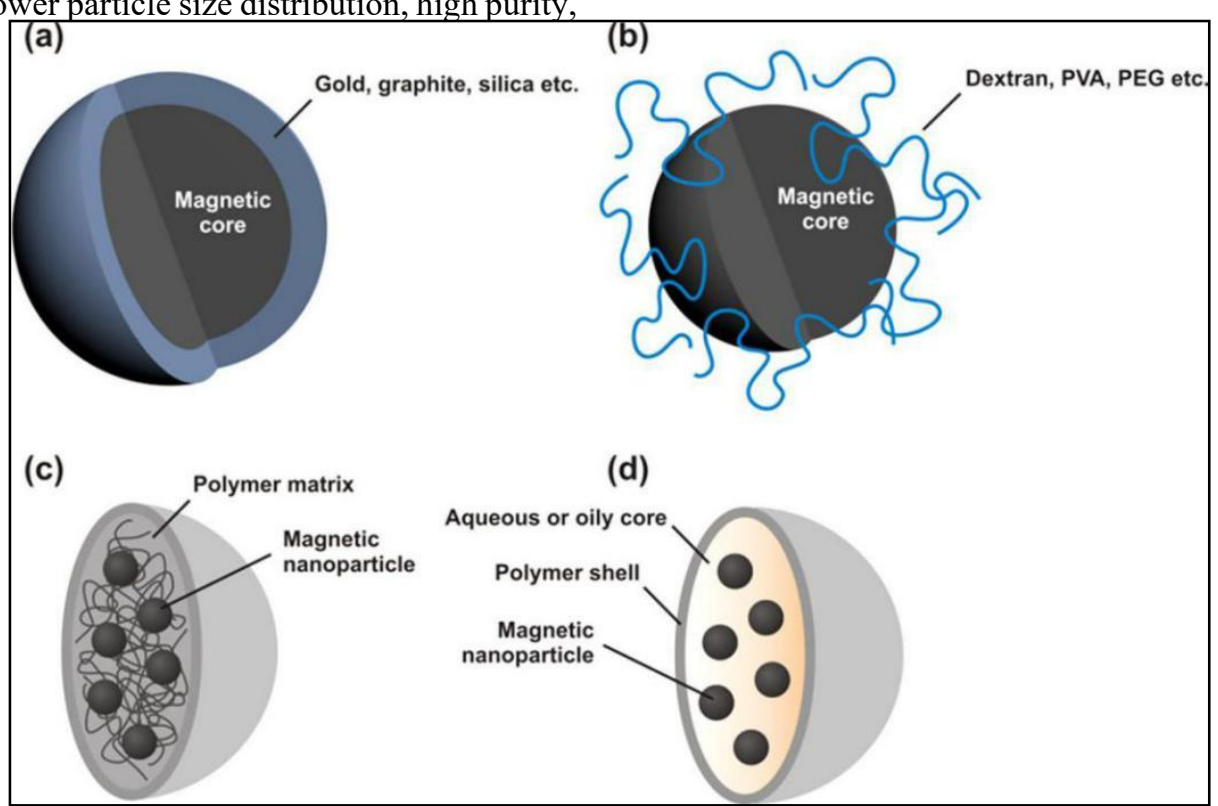

Fig. 1. Structure of magnetic nanomaterials coated with the four materials ${ }^{[6]}$

Currently, monomer polymerization is widely used in the preparation of magnetic nanospheres. A large number of scholars prepared magnetic nano-microsphere which has high saturation magnetization. Surface modification carried out on the microspheres can help improve the stability, and can make the material functional, which improves the target detection rate. Therefore, the combination of material in the future further research and application, magnetic nano microsphere surface nanoparticles. Meanwhile, it is necessary to develop more modification method, coat the magnetic particles, improve the chemical stability, prevent oxidation, and give its special function.

Magnetic nanoparticles (MNPs) have a core-shell structure that uses magnetic nanoparticles as the core to be wrapped with favorable materials. Nanoscale microspheres have strong adsorption properties because of their huge specific surface area. $\mathrm{Fe}_{3} \mathrm{O}_{4}$ is commonly used as magnetic cores. Natural biomaterials and polymers are made to be nanospheres' shells. Figure 1 shows the magnetic nanomaterial junction coated by inorganic (a), organic material (b), nanoparticle (c) and nanocapsules (d) structure. modification which improves the detection efficiency will become an important development trend.

\section{Specific methods for the application of magnetic nanomaterials in practical detection}

\subsection{Magnetic solid-phase extraction (MSPE)}




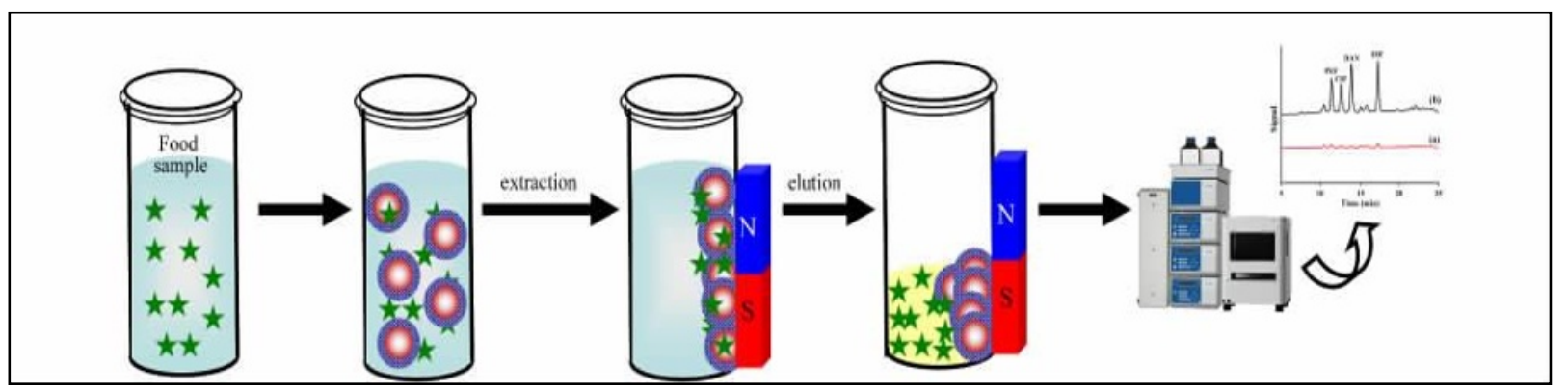

Fig. 2. MSPE flow chart ${ }^{[7]}$

Magnetic Solid-phase extraction (MSPE) ${ }^{[7]}$ is an efficient and easy method of separation. When we put the magnetic nanoparticles or micro ball evenly dispersed in the sample solution, the target material under test is adsorbed. When joining the external magnetic field, target materials with magnetic nanomaterials were separated. The target substance is then eluted with a solvent. Thus the enriched substance to be measured is obtained. And magnetic nanoparticles can be recycled. Compared with traditional SPE, MSPE has the following advantages. Firstly, MSPE does not use the SPE column, and there is no problem with long consumption and easy blockage when passing through the column. Secondly, only a small amount of magnetic nanomaterials can be used to adsorb the substance to be measured in a short time, and the consumption of organic solvent is very small. Magnetic nanomaterials can also be recycled. Thirdly, the magnetic response characteristic of magnetic nanomaterials is directly used to quickly separate the substance to be measured, which eliminates the steps of centrifugation and filtration. Lastly, the whole process is automated, and the magnetic nanomaterials have high efficiency and accuracy due to its high adsorbability and selection specificity.

\subsection{Magnetic sensor}

The magnetic induction effect of magnetic nanomaterials greatly improves the detection efficiency of biosensors. The combination of magnetic nanomaterials with light and heat can realize real-time on-site monitoring and in vivo detection. At present, electrochemical sensors, electrochemical sensors, and electrochemiluminescence immunoassay sensors have developed rapidly and been widely used.

The immune sensor is formed by combining an antibody with magnetic nanomaterials, which can specifically detect the object to be tested with high sensitivity, good selectivity, and fast detection speed. Electrochemical sensors utilize the high electro-catalytic effect of magnetic nanomaterials to enhance the boundary response and electron conduction ${ }^{[8]}$, which is faster than the detection speed of immune sensors. Electrochemical Immunosensor combines the characteristics of the electrochemical sensor and immunosensor, and has better stability and reproducibility.

However, the sensors described above as well as optical sensors require more complex pretreatment, so these methods are greatly affected by the medium. At present, magnetic relaxation switch nanosensors are further studied by scholars. This method does not require complicated pre-processing steps and can even achieve background interference-free detection. This technique uses magnetic contrast material to change the relaxation time, and the relaxation time T2 changes when the state of magnetic nanomaterials changes from dispersion to aggregation $^{[9]}$. Therefore, by detecting the change of $\mathrm{T} 2$, the quantitative determination of the target can be achieved. Currently, magnetic relaxation switch nanosensor has been applied to food safety detection, such as heavy metal ions, small molecular substances such as kanamycin in milk, and pathogenic bacteria such as enteric Salmonella ${ }^{[9]}$. In the future, more attention should be paid to the research of magnetic sensors with higher selectivity, high flux, and high sensitivity.

\section{Applications of magnetic nanomaterials in actual detection}

\subsection{Pesticide detection}

Pesticide residue is one of the biggest problems in food safety at present, and it also seriously affects the ecological environment. Therefore, accurate, sensitive, rapid, and high-throughput pesticide residue detection technology is urgently needed. The following scholars used ionic liquids, polymer materials, metal-organic framework materials, and other modified magnetic nanomaterials to obtain efficient detection of food pesticide technology.

$\mathrm{Amir}^{[10]}$ et al. prepared polyoxometallate liquid magnetic hybrid particles (BeW12O40-ILSCcfNPs). By combining GC-FID to determine OPPs in water samples and juice, they got the LOD was $0.02-0.06 \mathrm{ng} / \mathrm{L}$. In the experiment, OPPs separation and enrichment were completed in 5 minutes, which could be repeated at least 10 times. Meseguer-Lloret ${ }^{[11]}$ et al. copolymerized glycidyl methacrylate (GMA) and ethylene glycol dimethacrylate (EDGMA) to obtain (POLY-(GMA-co-EDMA), and then reacted 3-Aminopropyltrimethoxysilane with the epoxy group to silanize the copolymer, making $\mathrm{Fe} 3 \mathrm{O} 4$ surface hydroxyl group combined with silylation group to prepare magnetic nanomaterials. In the experiment, the magnetic nanomaterials can be used to efficiently detect 3 organophosphorus pesticides in water samples, which can be repeated at least 50 times. Liang ${ }^{[12]}$ et al. prepared $\mathrm{Fe}_{3} \mathrm{O}_{4} @ \mathrm{SiO}_{2}-\mathrm{GO}$ magnetic nanoparticles, adsorbed, and reacted with $\mathrm{Gr}^{3+}$ to obtain magnetic metal-organic framework composite $\mathrm{Fe}_{3} \mathrm{O}_{4} @ \mathrm{SiO}_{2}-\mathrm{GO} / \mathrm{MIL}-101$ (Cr), 
and realized rapid separation and enrichment and HPLC-UV detection of seven triazine herbicides in rice samples. And LOD is $0.010^{-} 0.080 \mu \mathrm{g} / \mathrm{kg}$.

\subsection{Veterinary drug detection}

Nowadays, China's animal husbandry industry is booming, with great demand for animal husbandry related industries. Veterinary drugs have been widely used to prevent animal diseases, promote growth and reproductive capacity, etc. However, veterinary drug residues in animal products will seriously harm human health and the ecological environment. There have been many reports on the application of MSPE in veterinary drug residue detection.

Zhang Xiaomin ${ }^{[13]}$ prepared graphene oxide magnetic nanocomposites and used MSPE to adsorb and extract. And they determine the residue of phenol in honey samples by high-performance liquid chromatography. The detection limit was $0.02 \mu \mathrm{g} \cdot \mathrm{L}^{-1}$, and the recovery was $87.3 \%-92.0 \%$. Wang Yaqun ${ }^{[14]}$ et al. prepared $\mathrm{Fe}_{3} \mathrm{O}_{4}$ magnetic nanoparticles, and then synthesized $\mathrm{Fe}_{3} \mathrm{O}_{4} @ \mathrm{SiO}_{2} @ \mathrm{MIP}$ molecular imprinted polymers by ultrasonic-assisted and precipitation polymerization. Three kinds of tetracycline residues in meat and milk were determined by HPLC. In the experiment, various antibiotic residues were detected in the samples within 15 minutes, and the detection limits of tetracycline, tetracycline, and aureomycin were 7.84, 9.61 and 11.93 $\mathrm{ng} / \mathrm{mL}$, respectively, with good reproducibility.

\subsection{Heavy metal detection}

With the development of industry, heavy metal ion pollution in the environment is becoming more and more common. When these pollutants circulate into crops, aquatic plants, and animals, and continue to accumulate in the food chain, there will be a bad impression on human health. Therefore, the detection of heavy metals is a key part of food safety quality detection. At present, many scholars have developed magnetic nanomaterials with specific recognition, which greatly improves the extraction efficiency.

NZVI (Nanoscale Zero Iron) is the obtained magnetic nanomaterials through grain refinement, and studies show that it can effectively adsorb heavy metals and organic pollution in water samples. Yan ${ }^{[15]}$ et al. compared the adsorption capacity of nZVI and micron grade zero-valent iron to chromium ions in water samples and found that the adsorption capacity of nZVI was much higher than that of the latter. Ito ${ }^{[16]}$ et al obtained FeS magnetic nanoparticles using desulphurized vibrio bacteria. After testing $\mathrm{As}^{3+}$, $\mathrm{Cd}^{2+}, \mathrm{Hg}^{2+}, \mathrm{Pb}^{2+}$ in the water samples, the results showed that the magnetic nanomaterials has good adsorption and could remove more than $95 \%$ of heavy metals.

\subsection{Contamination detection of additives and synthetic pigments}

With the development of the food industry, more and more diverse and functional additives and synthetic pigments have been developed. According to national standards, food enterprises should strictly follow the requirements of the use of additives and pigments. But in recent years, there are still many cases of pollution caused by abuse, and excessive use of these chemicals is highly teratogenic and carcinogenic. At present, some scholars have applied MSPE or MSPE combined with fluorescence spectrophotometry in the detection of these substances and prepared specifically modified magnetic nanomaterials to greatly improve the detection rate.

Chai Weibo ${ }^{[17]}$ et al. prepared $\mathrm{Fe}_{3} \mathrm{O}_{4} @$ PDA NPs magnetic nanocomposite material to detect synthetic pigments of amaranth red, ponceau, sunset yellow and seducing red in the beverage, and the results showed that the amount of magnetic nanomaterials adsorption was less and the detection sensitivity was higher than other detection methods, and the detection limit was $0.20-0.25$ $\mu \mathrm{g} \mathrm{L}^{-1}$. $\mathrm{Fe}_{3} \mathrm{O}_{4} @ \mathrm{SiO}_{2} @[\mathrm{OMIM}] \mathrm{PF} 6$ was prepared by Almojtaba Abd Alkhalig Ahmed Bakheet ${ }^{[18]}$ for MSPE. Rhodamine B was enriched, separated, and analyzed by fluorescence spectrophotometry. The detection limit was $0.40-140.0 \mu \mathrm{gL}^{-1}$ with a correlation coefficient of 0.9998 . And the method can reuse at least 10 times.

\subsection{Detection of pathogenic bacteria and mycotoxin}

It is reported that 76 million people in the United States are infected with foodborne diseases each year, of which 32,500 are hospitalized and 5,000 are fatal, and food-borne pathogens account for $19.4 \%^{[19]}$. Data show that in 2006, there were 594 outbreaks of foodborne diseases in Chinese foodborne disease surveillance areas, with a total of 13,849 cases and 67 deaths, of which $48.3 \%$ were caused by foodborne pathogens ${ }^{[20]}$. In recent years, magnetic nanomaterials with high sensitivity and specificity have been widely used in the detection of pathogenic bacteria and mycotoxins.

Wang $\mathrm{Jie}^{[21]}$ prepared an Immunol functional $\mathrm{Au}-\mathrm{Fe}_{3} \mathrm{O}_{4}$ magnetic nanoprobe for the determination of Staphylococcus aureus. This method has high sensitivity and specificity and compared with traditional detection methods such as multiplex PCR, it has lower target concentration and higher sensitivity. Sun Cheng ${ }^{[22]}$ prepared positively charged amino functionalization of magnetic nanoparticles to separate and enrich bacteria, using multiplex PCR techniques to detect Staphylococcus aureus in food, single Listeria, Listeria monocytogenes, and Salmonella enteritidis. The capture rate is high and the method can be used in the detection of milk, watermelon, pickles, braised pork. Guo Ting ${ }^{[23]}$ et al. prepared magnetic nanomaterials and used fluorescence sensor to determine aflatoxin M1 in milk with a detection limit of $0.02 \mu \mathrm{g} / \mathrm{L}$ and recovery rate of $82.5 \%-102.3 \%$.

\section{Discussion and Prospect}

The research and development of new functional magnetic nanomaterials have become one of the main development trends. Besides, the functionalization of magnetic nanomaterials combined with traditional detection 
technology has greatly improved the efficiency of actual detection, which should be the focus of future research. Through a review of the application of functional magnetic nanomaterials, the advantages of functional magnetic nanomaterials in application are analyzed and summarized as follows.

Magnetic nanomaterials tend to agglomerate, resulting in poor adsorption selectivity and reducing enrichment effect. However, the modified magnetic nanomaterials can solve this problem and have the following advantages. 1) Superparamagnetic, high capturing capability. 2) Adsorption specificity and selectivity. 3) Control particle size and enhance dispersion ability. 4) Enhance selective adsorption enrichment and sensitivity. Reduce detection limit and make detection faster. 5) Biocompatibility, not easy to degrade and easy to regenerate. 6) Make physical and chemical properties stable and solve the problem of agglomeration and poor dispersion.

Detection technologies based on magnetic nanomaterials, such as magnetic resonance relaxation transformation and low game combined with magnetic relaxation, MSPE, nanometer sensor switch, are more efficient than traditional techniques such as the multiple PCR, enzyme-linked immunoassay (ELISA), thin-layer chromatography (TLC), gas chromatography (GC), high-performance liquid chromatography (HPLC) and temperament (GC-MS) ${ }^{[1]}$. And they have economic, efficient, accurate, fast, and convenient features. Meanwhile, there still needs improvement to functional magnetic nanomaterials and related detection technologies.

Magnetic nanomaterials are easier to be oxidized and agglomerated than other nanomaterials particles. Now we have solved this problem with surface modification and functionalization. However, the magnetic responsivity of the modified magnetic nanomaterials is somewhat affected, so it is necessary to further solve the problem of how to maintain the magnetic responsivity of functional magnetic nanomaterials in the future. During the detection of functionalized magnetic nanomaterials, they may be affected by the sample matrix, resulting in the shedding of modifying groups and lower enrichment efficiency. Therefore, we need to develop functional magnetic nanomaterials that are more stable and not affected by the matrix to meet various complex detection conditions. Currently, relevant technologies of functional magnetic nanomaterials detection have not been automated, so the automatic research and development of devices like MSPE is also a future development direction. The preparation process of functional magnetic nanomaterials is relatively complex. Currently, we have not been able to realize the mass production and continuous production of functional magnetic nanomaterials. Therefore, we still need to develop the synthesis technology of functional magnetic nanomaterials from the perspective of more practical factory production, to obtain large quantities of materials with good economic and reproducibility. The preparation process, use, and recovery process of modified magnetic nanomaterials may cause secondary environmental pollution. Therefore, in the selection of raw materials for functional groups, we need to choose more green and environment-friendly materials. Most studies on functional magnetic nanomaterials tend to optimize the enrichment and adsorption conditions, and few researchers explore the working mechanism of the material. Therefore, physical and chemical characterization techniques and theoretical data models can be used to study and improve the performance of functional magnetic nanomaterials.

Although great progress has been made on the functionalized magnetic nanomaterials for food testing, with the food industry rapid development, as well as relevant pesticides, veterinary drugs, such as research and development of fast, and the worsening ecological environment, we must be more strict and efficient in terms of food quality and safety testing technology. Therefore, in the future, we need to further study the preparation, technical application, performance improvement, and functional diversification of functional magnetic nanomaterials, helping magnetic nanomaterials have more extensive applications. In the future, surface modification of magnetic nanomaterials improving functional diversity will become an important development trend. In the research and development of relevant technologies, we need to step forward to technologies with higher selectivity, high throughput, and high sensitivity, and make continuous improvement achieved more convenient in-situ real-time monitoring. MSPE and other advanced technologies are combined with various analytical technologies to develop more miniaturized, automated, high-throughput, online detection methods.

\section{Conclusion}

Functional magnetic nanomaterials have eliminated the limitations of the application of magnetic nanomaterials and solved several problems of traditional detection techniques, having been widely used in the field of food detection. MSPE, magnetic sensor and other technologies are more efficient applications of the material. The material has been widely used in food pesticides, veterinary drugs, heavy metals, additives and synthetic pigments, pathogenic bacteria and mycotoxins. In the future, people still need to conduct more in-depth research on the stability of materials, physical and chemical properties. In terms of application, they also need to realize mass production of materials, develop more automated technologies, and research and develop more green and environmental friendly materials.

\section{Acknowledgment}

I would like to thank Miss Zhang Jingjing for her guidance in my thesis writing. I would like to thank my mother for giving full support to my scientific research and professional research. I would like to thank my friend Gaoda for sharing literature search and other methods in my thesis writing, as well as Zeng Ruowei for encouraging me in my writing. 


\section{References}

1. Chu H Q, Lu Y F. progress in preparation and application of functional nanomaterials in food safety testing [J]. Analytical Chemistry, 2010, 38(03):442-448. (in Chinese)

2. Wang Y J. Preparation of magnetic nanoparticles and their application in the biomedical field [J]. Shandong Chemical Industry, 2018, 047(022):91-92. (in Chinese)

3. Chend G X, Shen F, Yang L F, et al. On properties and structure of the AOT-water-isooctane reverse micellar microreactor for nanoparticles, Mater Chem Phys, 1998, 56( 2): 97-101.

4. Rockenberger J, Scher E C, Alivisatos A P. J. Am. Chem. Soc., 1999, 121( 49): 11595-11596.

5. Pileni M P. Nature Materials, 2003, 35( 2): 145-150.

6. Reddy L H, Arias J L, Nicolas J, et al. Magnetic nanoparticles: design and characterization, toxicity and biocompatibility, pharmaceutical and biomedical applications[J]. Chemical Reviews, 2012, 112: 5 818-5 878.

7. Gao Q, Luo D, Ding J, et al. J Chromatogr A, 2010, 1217( 35):5602.

8. Vodinh T, Liu Y, Fales A M, et al. SERS Nanosensors and Nanoreporters: Golden Opportunities in Biomedical Applications[J]. Wiley Interdisciplinary Reviews: nanomedicine \s\& । snanobiotechnology, 2015, 7 ( 1): 17-33.

9. Wang $\mathrm{R}, \mathrm{Xu} \mathrm{Z}$, Chen Yanqiu, Chen Maolong, zhu Yingyue, Ding Li, Cheng Yunhui. Research progress of magnetic relaxation switch nanometer sensor in food safety detection [J]. Food Science, 2020, 41(09):263-268. (in Chinese)

10. Amiri A, Saadati-Moshtaghin H R, Zonoz F M. Mikrochim Acta, 2018, 185( 3): 176.

11. Meseguer-Lloret S, Torres-Cartas S, Catalá-Icardo M, Simo-Alfonso E F. Herrero-Martinez J M. Anal Bioanal Chem, 2017,409( 14) : 3561.

12. Liang L, Wang X, Sun Y, Ma P, Li X, Piao H, Jiang Y, Song D. Talanta, 2018, 179: 512.

13. Zhang $\mathrm{X}$ M. Application of MAGNETIC solid-phase extraction/high-performance liquid chromatography in the analysis of veterinary drug residues in food [D]. Shanxi Normal University, 2019. (in Chinese)

14. Wang Y Q, Pan D D. Magnetic imprinted solid-phase extraction of tetracycline antibiotics in food $[\mathrm{J}]$. Food Industry Science and Technology, 2015, 36(18): 53-58. DOI:10.13386/ J.ISsn1002-0306.2015.18.002. (in Chinese)

15. Yan W, Herzing A A, Kiely C J, et al. Nanoscale zero-valent iron (nZVI): Aspects of the core-shell structure and reactions with inorganic species in water[J]. Journal of Contaminant Hydrology, 2010, 118(3-4):96-104.

16. Ito $\mathrm{D}$, Miura $\mathrm{K}$, Ichimura $\mathrm{T}$, et al. Removal of As, $\mathrm{Cd}, \mathrm{Hg}$, and $\mathrm{Pb}$ ions from solution by adsorption with bacterially-produced magnetic iron sulfide particles using high gradient magnetic separation[J]. IEEE Transactions on Applied Superconductivity, 2004, 14(2):1551-1553.

17. Chai Weibo, Wang H J, Zhang Y, et al.Preparation of polydopamine coated magnetic nanoparticles for dispersive solid-phase extraction of water-soluble synthetic colorants in beverage samples with HPLC analysis[J]. Talanta, 2016,149:13-20.

18. Almojtaba Abd Alkhalig Ahmed Bakheet. Analysis of food pigments by magnetic solid-phase extraction (MSPE) using ionic liquid coated magnetic nanoparticles [D]. Yangzhou University, 2018. (in Chinese)

19. MEAD P S, SLUTSKER L, DIETZ V, et al. Food-related illness and death in the United States[J]. Emerging Infectious Diseases, 1999, 5: 607-625.

20. Wang Z T, Chen Y, GUO Y C, et al. Surveillance data analysis of foodborne disease outbreaks in China in 2006 [J]. Health Research, 2006, 39(3): 331-334. (in Chinese)

21. Wang J. Staphylococcus aureus T 2 model low field magnetic resonance detection method [D]. Shanghai Normal University, 2020. (in Chinese)

22. Sun C. Enrichment of amino-functionalized magnetic nanoparticles combined with multiplex PCR for the detection of foodborne pathogenic bacteria [D]. Huazhong Agricultural University, 2019. (in Chinese)

23. Guo T, Lin S F, Ma L, Tan H X, Zhang Y H. detection of aflatoxin M_1 in milk by fluorescence sensor based on magnetic nanometer material and aptamer [J]. Food and fermentation industry, 2019, 45(05):218-223. (in Chinese) 